\title{
Allogeneic hematopoietic stem cell transplantation and solid organ transplantation in the same patient
}

\author{
Tapani Ruutu' ${ }^{1}$ Christian Koenecke ${ }^{2}$ and Grzegorz W. Basak ${ }^{3}$ \\ ${ }^{1}$ Helsinki University Central Hospital, Comprehensive Cancer Center, Stem Cell Transplantation Unit, Helsinki, Finland; \\ ${ }^{2}$ Hannover Medical School, Department of Hematology, Hemostasis, Oncology and Stem-Cell Transplantation, Hannover, \\ Germany; \\ ${ }^{3}$ The Medical University of Warsaw, Department of Hematology, Oncology and Internal Diseases, Poland
}

Tapani Ruutu, Helsinki University Central Hospital, Biomedicum 2 C, POB 705, FIN-00029 HUS, Helsinki, Finland
Phone: +358-50-555-12-79

Fax: +358-9-4717-15-06

E-mail: tapani.ruutu@hus.fi

\section{Summary}

The present review is largely based on two surveys on patients who have undergone both an allogeneic hematopoietic stem cell transplantation (HSCT) and a solid organ transplantation (SOT), carried out by the European Society for Blood and Marrow Transplantation (EBMT). Some additional references to the literature are included. From these sources of data it can be summarized that SOT represents a valuable treatment strategy in HSCT recipients who develop an organ failure. In stringently selected young patients, the overall and organ survivals appear to be comparable to patients undergoing SOT for other causes. Complications, such as infections and graft rejection are frequent but usually manageable. Thus, SOT offers a viable therapeutic option for selected patients with single organ failure after HSCT. Also, selected SOT recipients suffering from hematologic disorders may benefit from allogeneic SCT and experience long-term survival without loss of organ function.

\section{Keywords}

allogeneic hematopoietic stem cell transplantation, renal transplantation, liver transplantation, heart transplantation, combined transplantations.

\section{Introduction}

Solid organ transplantation (SOT) is widely used for the treatment of end-stage organ insufficiency. About 30000 SOTs were performed in the European Union in 2011, comprising approximately 19000 kidney, 7000 liver and 2000 heart and other (including combined) transplants [3]. Allogeneic hematopoietic stem cell transplantation (HSCT) is the only cure or the most effective treatment for a large number of malignant and non-malignant diseases of the lympho-hematopoietic system with an otherwise poor prognosis. At present, the number of allogeneic HSCTs in Europe is approximately 15000 per year [8]. With these large num- bers of patients, the likelihood of coincidental occurrence of disorders potentially indicating SOT and HSCT in the same patient is increasing. Such transplantation combinations can be problematic for many reasons.

Allogeneic HSCT is an intensive treatment which causes frequently organ injury and failure, resulting in a potential indication for a SOT. The mechanisms of organ injury include cytotoxic drugs and radiotherapy in the pretransplant conditioning, organ-toxic agents in supportive care, and immunological mechanisms. The organs most often affected are kidney, liver, lung and heart. Factors that may complicate a SOT in this setting are the fragility of the patient as a consequence of the toxicity of HSCT, immunosuppressive treatment for 
the HSCT which may not be optimal for the SOT, potential immunological problems caused by tissue type differences between the recipient, the hematopoietic graft and the solid organ graft, and infections. In SOT recipients, the long-lasting immunosuppressive medication may affect the function of organs, and increase the risk of infections. SOT can be associated with development of hematological disorders comprising aplastic anemia, post-transplant lymphoproliferative disease, acute myeloid leukemia and myelodysplastic syndromes [1] which may be a potential indication for allogeneic HSCT.

As the clinical situation is often found to be complicated and the risk of treatment failure regarded as high, the numbers of SOTs to HSCT recipients and those of HSCTs to SOT recipients have been low as compared to the number of patients who would have a potential indication for such treatment. Until recent years the literature of such transplantations consisted mainly of case reports as reviewed by Basak et al. [1], Chiang and Lazarus [2], Koenecke et al. [7], with obvious risk of publication bias. As the results of such combined transplantations were not well known, the European Society for Blood and Marrow Transplantation (EBMT) carried out two surveys to collect the experience in Europe and to analyze the outcome. One survey evaluated the experience with SOT in HSCT recipients, the other HSCTs carried out in SOT recipients.

\section{EBMT surveys}

In the EBMT survey of SOTs carried out in HSCT recipients by Koenecke et al [7], 107 centers with the total of 67000 allogeneic HSCTs carried out between 1984 and 2007 participated. Among them, 28 centers reported having carried out a total of 45 SOTs in 40 patients for organ failure after HSCT. The transplantations comprised 15 renal, 15 liver, 13 lung grafts, 1 heart, and 1 skin transplantation. In approximately $2 / 3$ of the HSCTs, they were performed for malignant hematological disease $(n=28)$, the other patients had aplastic anemia, or an inherited disorder ( $n=6$ for both). In most cases, the donor had been a sibling, and bone marrow has been transplanted. Almost all patients had received a standard full-intensity conditioning. Twenty-two patients had developed grade II or higher acute GVHD and 23 patients, extensive chronic GVHD before the SOT. This was the cause for SOT in six patients receiving liver transplants, and in 13 patients undergoing lung transplantation. In all other patients, clinically significant GVHD resolved before the SOT. Median age at the time of the renal transplantation was 35 (range 8-64) years, for liver transplantation, 20 (1-56) years, and for lung transplantation, 32 (11-55) years. The median time between HSCT and renal transplantation was 84 (range 8-188) months, that in liver transplantations 34 (range 1-137) months, and in lung transplantations, 69 (range 7-196) months. In renal transplantations following HSCT, the most common cause was drug- or radiation-induced kidney failure. Patients receiving liver transplantation could be divided in two groups, early and late transplantation after HSCT. Early liver transplantation (up to six months post-HSCT) was performed due to acute liver failure caused by veno-occlusive disease (VOD) or acute GVHD, late transplantation (usually, 2-5 years after HSCT) was mostly carried out because of chronic liver-GVHD or cirrhosis. The indication for lung transplantation was bronchiolitis obliterans (BOS)/ chronic GVHD in all these patients. The overall 5 -year survival after SOT was 78\%, in renal transplantations, $100 \%$, in liver transplantations, $71 \%$ and in lung transplantations, $63 \%$. None of the renal transplantation patients was on dialysis at the end of the follow-up. The overall 2-year incidence of SOT failure was $16 \%$ (20\% in patients with GVHD preceding SOT versus 7\% in GVHD-free cases). Graft rejection occurred in approximately $30 \%$ of the patients with each type of transplantation, but it could be usually treated. Two patients with renal transplantation, 2 with liver transplantation and 4 with lung transplantation experienced graft failure. The relapse incidence of the underlying malignant disease was $4 \%$ at 5 years after the SOT. Six patients received the SOT from the original HSCT donors (4 kidneys, 1 liver, 1 lung transplantation). All of them, except of the lung transplantation patient, were alive at the time of the analysis.

In the course of EBMT survey by Basak et al [1], with 101 centers participating, 18 centers reported a total of 31 HSCTs in 28 SOT recipients. Thirteen of the patients have received a liver transplantation, 12 patients received renal grafts, and three underwent heart transplantation. Malignant hematological disease was the reason for HSCT in 22 of the 28 patients, the most common indications being acute myeloblastic leukemia (AML) in 8 cases, acute lymphoblastic leukemia (ALL) and chronic myeloid leukemia (CML) in 4 cases each. The diagnosis leading to HSCT was known before the SOT in 8/28 patients (AML, ALL, CML, chronic granulomatous disease, primary immunodeficiency, severe aplastic anemia). The patients were young, with median age at the HSCT of 25 (1-62) years. The median time from SOT to HSCT was 37 (1-315) months. The sources of hematopoietic graft were as follows: peripheral blood stem cells in 21, bone marrow in 9, and the both in one patient. The donor was a HLA-identical sibling in 12 , a matched unrelated donor in 14, and haploidentical, in 4 cases. Seventeen patients received myeloablative conditioning. In the rest of this group, intensity of the conditioning was reduced. Ex vivo T-cell depletion was carried out in five cases. Successful engraftment was registered in 25/26 evaluable patients. Eight patients developed grade II-IV acute GVHD. Chronic GVHD occurred in seven patients. Despite commonly advanced stage of the underlying disease at the time of HSCT, the relapse rate was low (22\%). With a 5-year follow-up, solid organ graft failure occurred in 9 of 31 patients, i.e., in 5/13 (38\%) of the renal transplantations, 3/15 (20\%) of the liver transplantations, and in one of the three heart transplantations. Five of the nine failures were defined as graft rejection. The median time to graft failure was 1.8 months, with a range of 0 to 131 months. Transplant-related mortality at three months was $25 \%$. Infection was the most common cause of death. The overall survival at five years after allogeneic HSCT was $40 \%$ for the entire group, $51 \%$ for liver transplant recipients and $42 \%$ for renal transplant recipients. There were no significant differences in the outcomes between different types of HSCT donors or grafts, or the conditioning intensity. 


\section{Other clinical experience}

Numerous case reports have described successful liver, lung, kidney and heart transplantation after HSCT. Similarly, several case reports over the last 20 years have detailed encouraging results of allogeneic HSCT in solid organ transplant recipients. These experiences have been summarized by Chiang and Lazarus [2], Koenecke et al [7], and Basak et al [1].

Holm and coworkers $[5,6]$ presented a survey from the Nordic countries about lung transplantations carried out for BOS after allogeneic HSCT. Thirteen patients were reported. The indication for the HSCT had been a malignant hematological disease in all but two patients. All had clinical chronic GVHD. The median time from the HSCT to the lung transplantation was 8.2 (range 0.7-16) years. The median age at the lung transplantation was 34 (16-55) years. With a median follow-up time from the lung transplantation of 4.2 years, the survival of the patients was $90 \%$ at one year, and $75 \%$ at 5 years. Of the two deceased patients, one died with a relapse of underlying disease, and one of infection. These results were compared to the data obtained from the Scandiatransplant registry and did not differ from the general cohort of lung transplant patients. Four patients developed BOS in the transplanted lung; in one case a retransplantation was carried out.

Vogl et al. [9] reported a cohort of seven patients who received lung transplantation for BOS after allogeneic HSCT. In this cohort there was a case fatality rate of $57 \%$, and median survival was 24 months after the lung transplantation. In this cohort the interval between HSCT and lung transplantation was much shorter than in the study of Holm et al. [5], with a median of 18 (6-120) months.

\section{Discussion}

Although a considerable number of reports describing SOT following HSCT have been published [2, 7], the number of such transplantations has been small in relation to the frequent problems of organ injury and failure seen in clinical practice. There are apparently many reasons to this, including the often complicated clinical situation, poor general condition of the patient, and possibly a certain resistance of clinicians to consider such treatment approach, but also insufficient information of such transplantations results. The described recent publications show that the outcomes can be quite good. In a survey by Koenecke et al. [7], the 5-year survival of patients receiving a renal transplantation was $100 \%$, with no patient being on dialysis. The 5-year survival for liver transplantation was $71 \%$ and that for lung transplantation $63 \%$. These figures are nor markedly different from the outcomes in general SOT populations. It is clear that the patient populations in dual transplantations have been selected, with young patient age, benign diseases somewhat overrepresented, and underlying malignant disease under good control, but the results show that SOT carried out for organ failure after HSCT is a feasible treatment option in carefully selected patients. There is no indication that prolonged immunosuppressive treatment due to the SOT would significantly increase the risk of disease relapse. However, it was noted by Koenecke et al. [7], that in a large proportion of the patients with SOTs following HSCT, they were carried out late, when the risk of relapse is already relatively low.

BOS is a serious and problematic complication of allogeneic HSCT, for which there is no effective treatment. Therefore, good results of lung transplantations for this disorder are encouraging. According to a report by Holm et al. [5], the 5 -year OS was $75 \%$, as compared with $63 \%$ in the study by Koenecke [7]. In contrast, the results presented by Vogl et al. [9] are showing that the median survival in a group of seven patients was only 24 months, and the case-fatality rate was $57 \%$. As discussed by Holm et al. [6], the timing of lung transplantation comprised the major difference between clinical data in their study and those by Vogl et al., i.e., the median time from HSCT having been 8.2 years in the Holm study versus 18 months in the Vogl study. This difference may reflect, at least in part, the general importance of patient selection: the patients undergoing SOT early after HSCT being more likely to be at a higher risk of failure because of rapidly progressing complications and a poorer general condition due to the recently performed HSCT.

The number of dual transplantations including cardiac transplantation published is small. Chiang and Lazarus [2] have identified only one patient presented. In the survey [7], one such patient was identified from EBMT centers, and three, in the survey by Basak [1]. Therefore, no general conclusions can be drawn on heart transplantations combined with HSCT.

In a HSCT recipient treated with SOT, the immunological conditions may be complicated. The immunosuppressive strategy applied in the two types of transplantations is somewhat different. These factors might affect the risk of solid organ graft rejection. In the survey of Koenecke et al [7], graft rejection was seen in 4/13 cases of renal transplantationsn, leading to kidney failure in two patients. These were treated with a second renal transplantation. In 2/14 liver transplantations, the rejection led to graft failure. In 2/10 lung transplantations rejection resulted in respiratory failure; in one case this was treated with repeated transplantation. Among the patients treated with lung transplantation in the study of Holm et al [6], no rejection causing organ failure took place. Therefore, also given the small numbers of patients, the incidences of graft rejection were not markedly different from those that can be seen in non-HSCT SOT patients. The occurrence or absence of GVHD did not significantly affect the outcome.

The number of reports of HSCT in SOT recipients in the literature is quite limited $(1,2)$. In such HSCTs the function and fate of the solid organ graft would be a concern. As shown in the survey by Basak et al [1], graft failure presents a distinct risk, as it was seen in $9 / 31$ patients. There was a renal graft failure in $38 \%$, liver graft failure in $20 \%$ and heart graft failure in $1 / 3$ of the patients. In approximately half of the cases, this was considered a result of rejection. There were no major problems with hematopoietic engraftment: with one exception, engraftment took place in all evaluable patients. 
There are many possible mechanisms of graft rejection in this transplant setting. An immune response can be directed against mismatched HLA molecules, because the hematopoietic graft is HLA-matched with the recipient rather than the solid organ. However, recent studies [4] have suggested that allogeneic HSCT can have a tolerogenic effect, allowing maintenance of a solid organ graft without immune suppression. Conditioning prior to allogeneic HSCT and infection may increase the immunogenicity of the organ graft by enhancing antigen presentation, increasing costimulatory signals, changing the properties of the vascular endothelium, and suppressing regulatory T-cell function. Whereas the majority of solid organ grafts are matched with the recipient based on their blood group, this is no require for HSCT donors. Thus, the majority of hematopoietic transplants are probably not matched with the solid organ graft, and this could affect the survival of the organ graft. Moreover, in most patients, the immunosuppressive regimen is changed after allogeneic HSCT from a regimen typical for SOT to an HSCT-specific regimen, which could be suboptimal in this situation.

In summary, SOT can represent a valuable treatment strategy in HSCT recipients who develop an organ failure. In stringently selected young patients, the overall and organ survivals appear to be comparable to patients undergoing SOT for other causes. Complications, such as infections and graft rejection are frequent but usually manageable. Thus, SOT offers a viable therapeutic option for selected patients with single organ failure after HSCT. Also, selected SOT recipients suffering from hematologic disorders may benefit from allogeneic HSCT and experience long-term survival without loss of organ function.

\section{Acknowledgements}

The authors declare no conflicts of interest.

\section{References}

1. Basak GW, Wiktor-Jedrzejczak W, Labopin M, Schoemans H, Ljungman P, Kobbe G, Beguin Y, Lang P, Koenecke $\mathrm{C}$, Sykora $\mathrm{KW}$, te Boome L, van Biezen A, van der Werf S, Mohty M, de Witte T, Marsh J, Dreger P, Kröger N, Duarte R, Ruutu T. Allogeneic hematopoietic stem cell transplantation in solid organ transplant recipients: a retrospec- tive, multicenter study of the EBMT. Am J Transplant 2015; 15: 705-14.

2. Chiang KY, Lazarus HM. Should we be performing more combined hematopoietic stem cell plus solid organ transplants? Bone Marrow Transplant 2003; 31: 633-42.

3. European Transplant Coordinators Organization. International donation and transplantation activity 2012. Available from: http://www.europeantransplantcoordinators.org.

4. Fandrich F. Cell therapy approaches aiming at minimization immunosuppression in solid organ transplantation. Curr Opin Organ Transplant 2010; 15: 703-8.

5. Holm AM, Riise GC, Hansson L, Brinch L, Bjortuft O, Iversen $\mathrm{M}$, Simonsen $\mathrm{S}$, Floisand Y. Lung transplantation for bronchiolitis obliterans syndrome after allo-SCT. Bone Marrow Transplantation 2013a; 48: 703-7.

6. Holm AM, Riise GC, Brinch L, Bjortuft O, Iversen M, Simonsen S, Floisand Y. Lung transplantation for bronchiolitis obliterans after allogeneic hematopoietic stem cell transplantation: unsolved questions. Transplantation 2013b; 96 : e21-e22.

7. Koenecke C, Hertenstein B, Schetelig J, van Biezen A, Dammann E, Gratwohl A, Ganser A, Schleuning M, Bornhäuser $M$, Jacobsen $N$., Kröger $N$, Niederwieser $D$, de Witte T, Ruutu T. Solid organ transplantation after allogeneic hematopoietic stem cell transplantation: a retrospective, multicenter study of the EBMT. Am J Transplant 2010; 10 : 1897-906.

8. Passweg JR, Baldomero H, Bader P, Bonini C, Cesaro S, Dreger P, Duarte RF, Dufour C, Falkenburg JHF, Farge-Bancel D, Gennery A, Kröger N, Lanza F, Nagler A, Sureda A, Mohty $M$ for the European Society for Blood and Marrow Transplantation (EBMT). Hematopoietic SCT in Europe 2013: recent trends in the use of alternative donors showing more haploidentical donors but fewer cord blood transplants. Bone Marrow Transplant advance online publication, February 2, 2015; doi:10.1038/bmt.2014.312

9. Vogl UM, Nagayama K, Bojic M, Hoda MAR, Klepetko W, Jaksch P, Dekan S, Siersch V, Mitterbauer M, Schellongowski P, Greinix HT, Petkov V, Schulenburg A, Kalhs P, Rabitsch W. Lung transplantation for bronchiolitis obliterans after allogeneic hematopoietic stem cell transplantation: a single-center experience. Transplantation 2013; 95: 623-28. 